LPTENS-94/25,

hep-th/9408076,

August 1994.

\title{
FROM WEAK TO STRONG COUPLING IN TWO-DIMENSIONAL GRAVITY
}

\author{
Jean-Loup GERVAIS \\ Jean-François ROUSSEL \\ Laboratoire de Physique Théorique de l'École Normale Supérieure円, \\ 24 rue Lhomond, 75231 Paris CEDEX 05, France.
}

\begin{abstract}
The strong coupling physics of two dimensional gravity at $C=7,13,19$ is deciphered, by building up on previous works along the same line. It is shown that chirality becomes deconfined. The string suceptibility is derived, and found to be real contrary to the continuation of the KPZ formula. Topological Liouville string theories (without transverse degree of freedom) are explicitely solved. Altough they involve strongly coupled gravity, they share many features with the standard matrix models.
\end{abstract}

\footnotetext{
${ }^{1}$ Unité Propre du Centre National de la Recherche Scientifique, associée à l'École Normale Supérieure et à l'Université de Paris-Sud.
} 


\section{Introduction}

As was already emphasized several times [1, 2], the operator approach to Liouville theory seems at present to be the only method applicable to the strong coupling regime. Indeed, although the Liouville exponentials loose meaning in the strongly coupled regime (since their operator product algebra involves operators and/or highest weight states with complex Virasoro weights), the general operator-family of their chiral components may still be used. The basic point is the derivation of truncation theorems [3, 1, 2] which show that, at Liouville central charges $C=7,13,19$, there exist subfamilies of the above chiral operators which form closed operator algebras, and are compatible with the reality condition of Virasoro weights. They are used to construct a new set of local fields which replace the Liouville exponentials. Since both sets are constructed out of the same free Bäcklund fields, they may be considered as related by a new type of quantum Bäcklund transformation, that connect the weak and strong coupling regimes of two-dimensional gravity. So far, however, and apart from the free Liouville string spectrum[ []], the physical properties of the strong coupling regime have remained mysterious. On the one hand, the truncation theorems follow from the quantum group structure of Liouville theory, and physics became a bit hidden by technicalities. On the other hand, the N-point functions of the Liouville string theories (with transverse degrees of freedom) seem to be beyond reach at the present time. In the present letter, we display the basic features (deconfinement of chirality, new expression for the string susceptibility) of the new set of local fields that replace the Liouville exponentials, in the strong coupling regime. The message will be that the derivation of the new features is very close to the previous weak-coupling one, once the new set of local physical fields is

established. Expanding upon the last section of ref. [2], we moreover compute the N-point functions of the strongly coupled topological models proposed earlier [2], which are Liouville string theories without transverse degrees of freedom. Some conjectural remarks are made as a conclusion.

\section{The weak coupling regime revisited}

Remarkably, the present operator method treats the weak and strong coupling regimes much on the same footing. Thus, as a preparation, we recall 
some basic points of the weak-coupling discussion[5, 6, 7]. Our conventions are to let

$$
Q_{L}=\sqrt{(C-1) / 3}, \quad Q_{M}=\sqrt{(25-C) / 3}
$$

where $C$ is the Liouville central charge. We call $\vartheta_{L}$, and $\bar{\vartheta}_{L}$ the two chiral components of the Bäcklund free field associated with the Liouville field $\Phi$. The building blocks of the quantum group approach to Liouville theory are chiral fields of the form

$$
\tilde{V}_{m \widehat{m}}^{(J \widehat{J})} \propto V_{-J-\widehat{J}}^{(J \widehat{J})} S^{J+m} \widehat{S}^{\widehat{J}+\widehat{m}}, \quad \tilde{\bar{V}}_{\bar{m}}^{(\bar{J} \widehat{\bar{m}})} \propto \bar{V}_{-\bar{J}-\widehat{\bar{J}}}^{(\bar{J} \widehat{\bar{J}})} \bar{S}^{\bar{J}+\bar{m}} \widehat{\bar{S}}^{\widehat{\bar{J}}+\widehat{\bar{m}}},
$$

The $V$ fields are functions of $z$, and the $\bar{V}$ fields functions of $\bar{z}$. The fields $V_{-J-\widehat{J}}^{(J \widehat{J})}$ are simple exponentials which may be directly re-expressed in terms of the Bäcklund free fields.

$$
V_{-J-\widehat{J}}^{(J \widehat{J})}=: \exp \left[\left(J \alpha_{-}+\widehat{J} \alpha_{+}\right) \vartheta_{L}\right]:, \quad \bar{V}_{-\bar{J}-\widehat{\bar{J}}}^{(\widehat{\bar{J}})}=: \exp \left[\left(\bar{J} \alpha_{-}+\widehat{\bar{J}} \alpha_{+}\right) \bar{\vartheta}_{L}\right]:
$$

$S, \bar{S}$, and $\widehat{S}, \widehat{\bar{S}}$ are the screening operators 6 associated with the two screening charges

$$
\alpha_{ \pm}=Q_{L} / 2 \pm i Q_{M} / 2 .
$$

The J's are quantum group spins, but we will not dwell upon this aspect. In practice they determine the weight of the $V$ operators which are of the type $(2 \widehat{J}+1,2 J+1)$ in the BPZ classification. We deal with irrational theories, since this will be the case in the strong coupling regime. Thus the range of $J$ and $\widehat{J}$ is unbounded. Concerning the Hilbert space, the Verma modules is of course charaterized by the same four quantum group spins. The highest weight states will be noted $|J, \widehat{J}>| \bar{J}, \widehat{\bar{J}}>$. Following the earlier studies [7], the chiral operators just written are noted with a tilde to emphasize their special normalization which is such that their fusing and braiding matrices are exactly equal to q-6j symbols. The corresponding highest-weight matrix elements of the chiral fields define the coupling constants [7, 2].

The local Liouville exponentials are given by [1], []

$$
e^{-\left(J \alpha_{-}+\widehat{J} \alpha_{+}\right) \Phi(z, \bar{z})}=\sum_{m, \widehat{m}} \widetilde{V}_{m \widehat{m}}^{(J, \widehat{J})}(z) \tilde{\bar{V}}_{m \widehat{m}}^{(J, \widehat{J})}(\bar{z})
$$


This form is dictated by locality and closure under fusion. For the following, it is important to stress that this expression only involves $V$ and $\bar{V}$ fields with equal quantum numbers $(J=\bar{J}, m=\bar{m}$, and so on), while $m$, amd $\widehat{m}$ are summed over independently. Thus the Liouville exponentials have zero conformal spins. On the other hand, it follows from the formulae just summarized that

$$
<J_{2}, \widehat{J}_{2}\left|\widetilde{V}_{m \widehat{m}}^{(J \widehat{J})}\right| J_{1}, \widehat{J}_{1}>\propto \delta_{J_{1}-J_{2}-m, 0} \delta_{\widehat{J}_{1}-\widehat{J}_{2}-\widehat{m}, 0},
$$

so that the Liouville exponential applied to a highest-weight state with $J=\bar{J}$, $\widehat{J}=\hat{\bar{J}}$ only gives states satisfying the same condition. Thus we may restrict ourselves to the subsector with zero winding number. We stress this well known fact, since this will not be true any more in the strong coupling regime. Let us next recall some main point of the derivation of the matrix model results in the present context following refs. [5, 2]. For $C>25, Q_{L}$ is real and $Q_{M}$ pure imaginary, so that $\alpha_{ \pm}$are real. The above formulae are directly useful. One represents matter by another copy of the theory summarized above, now with central charge $c=26-C$, so that $Q_{M}$ is its background charge. One constructs local fields in analogy with Eq.2.5:

$$
e^{-\left(J \alpha_{-}^{\prime}+\widehat{J} \alpha_{+}^{\prime}\right) \Phi^{\prime}(z, \bar{z})}=\sum_{m, \widehat{m}} \widetilde{V}_{m \widehat{m}}^{(J, \widehat{J})}(z) \widetilde{\bar{V}}_{m \widehat{m}}^{(J, \widehat{J})}(\bar{z}) .
$$

Symbols pertaining to matter are distinguished by a primeft. In particular $\Phi^{\prime}(z, \bar{z})$ is the matter field (it commutes with $\Phi(z, \bar{z})$ ), and $\alpha_{ \pm}^{\prime}$ are the matter screening charges

$$
\alpha_{ \pm}^{\prime}=\mp i \alpha_{\mp} .
$$

The correct dressing of these operators by gravity is achieved by considering the vertex operators

$$
\mathcal{W}^{J, \widehat{J}} \equiv e^{-\left((-\widehat{J}-1) \alpha_{-}+J \alpha_{+}\right) \Phi-\left(J \alpha_{-}^{\prime}+\widehat{J} \alpha_{+}^{\prime}\right) \Phi^{\prime}}
$$

which is an operator of weights $\Delta=\bar{\Delta}=1$. In particular for $J=\widehat{J}=0$, we get the cosmological term $\exp \left(\alpha_{-} \Phi\right)$. The three-point function was computed in refs [5, 2]. The corresponding product of coupling constants gives the correct leg factors after drastic simplifications. After that, one may follow the line of ref. $[\mathbb{8}$ and derive the higher point function. We will come back to this in the coming section.

\footnotetext{
${ }^{2}$ or, if more convenient by the index $M$.
} 


\section{The new local fields.}

At this point we turn to the strong coupling regime. Now $1<C<25$, and $Q_{L} Q_{M}$ are real. The screening charges $\alpha_{ \pm}$are complex and related by complex conjugation. Thus complex weights appear in general. There are two types of exceptional cases. The states $\mid J, J>($ resp. $\mid-J-1, J>$ ) have highest weights which are real and negative (resp. positive). One could try to work with the corresponding Liouville exponentials $\exp \left[-J\left(\alpha_{-}-\alpha_{+}\right) \Phi\right]$ $\left(\right.$ resp $\left.\exp \left[\left((J+1) \alpha_{-}-\alpha_{+}\right) \Phi\right]\right)$, but this would be inconsistent, since these operators do not form a closed set under fusing and braiding. Moreover, as is clear from Eqs.2.5, 2.6, they do not preserve the reality condition for highest weights just recalled. The basic problem is that Eq.2.5 involves the $V$ operators with arbitrary $m \widehat{m}$, while the reality condition forces us to only use $V$ operators of the type

$$
V_{m,+}^{(J)} \equiv \widetilde{V}_{-m m}^{(-J-1, J)}, \quad V_{m,-}^{(J)} \equiv \widetilde{V}_{m m}^{(J, J)} .
$$

Now is a good time to recall the truncation theorems which hold for

$$
C=1+6(s+2), \quad s=0, \pm 1 .
$$

First define the physical Hilbert space

$$
\mathcal{H}_{\text {phys }}^{ \pm} \equiv \bigoplus_{r=0}^{1 \mp s} \bigoplus_{n=-\infty}^{\infty} \mathcal{H}_{r / 2(2 \mp s)+n / 2}^{ \pm}
$$

where $\mathcal{H}_{J}^{ \pm}$denotes the Verma modules with highest weights $\mid \mp(J+1 / 2)-$ $1 / 2, J>$. The physical operators $\chi_{ \pm}^{(J)}$ are defined for arbitrary $2 J \in \mathcal{Z} /(2 \mp$ $s)$, and $2 J_{1} \in \mathcal{Z} /(2 \mp s)$. to be such that

$$
\chi_{ \pm}^{(J)} \mathcal{P}_{\mathcal{H}_{J_{1}}^{ \pm}}=\sum_{\nu \equiv J+m \in \mathcal{Z}_{+}}(-1)^{(2 \mp s)\left(2 J_{1}+\nu(\nu+1) / 2\right)} V_{m, \pm}^{(J)} \mathcal{P}_{\mathcal{H}_{J_{1}}^{ \pm}}
$$

\footnotetext{
${ }^{3}$ We could also introduce mixed operators of the type $V_{-m m}^{(J, J)}$ as done in ref. [1], or $V_{m m}^{(-J-1, J)}$, but they are not needed at present.

${ }^{4}$ By the symbol $\mathcal{Z} /(2 \mp s)$, we mean the set of numbers $r /(2 \pm s)+n$, with $r=0, \cdots$, $1 \pm s, n$ integer; $\mathcal{Z}$ denotes the set of all positive or negative integers, including zero.

${ }^{5} \mathcal{Z}_{+}$denotes the set of non negative integers.
} 
where $\mathcal{P}_{\mathcal{H}_{J_{1}}^{ \pm}}$is the projector on $\mathcal{H}_{J_{1}}^{ \pm}$. Denote by $\mathcal{A}_{\text {phys }}^{ \pm}$the set of fields $\chi_{ \pm}^{(J)}$, with $2 J \in \mathcal{Z} /(2 \mp s)$. The basic properties of the special values Eq.3.2 is the TRUNCATION THEOREM:

For $C=1+6(s+2), s=0, \pm 1$, and when it acts on $\mathcal{H}_{\text {phys }}^{+}\left(\right.$resp. $\left.\mathcal{H}_{\text {phys }}\right)$, the above set $\mathcal{A}_{\text {phys }}^{+}$(resp. $\mathcal{A}_{\text {phys }}^{-}$) is closed by braiding and fusion and only gives states that belong to $\mathcal{H}_{\text {phys }}^{+}$(resp. $\mathcal{H}_{\text {phys }}^{-}$).

Note that the operators $V_{m, \pm}^{(J)}$ themselves are not closed by fusing and braiding, contrary to the very specific combinations Eq.3.4. The proof is given in refs. 11, 2]. It follows from a neat mathematical property of the quantum group structure. In general, it is of the type [5] $U_{q}(\operatorname{sl}(2)) \odot U_{\widehat{q}}(\operatorname{sl}(2))$ with $h=\pi \alpha_{-}^{2} / 2, \widehat{h}=\pi \alpha_{+}^{2} / 2$, so that $h \widehat{h}=\pi^{2}$. The two quantum group parameters are thus related by duality. At the special values, one has, in addition $h+\widehat{h}=s \pi$. Then the q-6j symbols of the two dual quantum groups become equal up to a sign [2]. Using the orthogonality relation of the q-6j's this leads to the truncation theorems.

Next we construct local fields out of the chi fields. The braiding of the chi fields is a simple phase. On the unit circle, one has

$$
\chi_{ \pm}^{\left(J_{1}\right)} \chi_{ \pm}^{\left(J_{2}\right)}=e^{2 i \pi \epsilon(2 \mp s) J_{1} J_{2}} \chi_{ \pm}^{\left(J_{2}\right)} \chi_{ \pm}^{\left(J_{1}\right)}
$$

where $\epsilon= \pm 1$ is fixed by the ordering of the operator on the left-hand side in the usual way. From the spectrum of the $J$ 's, it follows that the phase factor is of the form $\exp (i \pi N / 2(2 \mp s))$, where $N \in \mathcal{Z}$. Thus, we have parafermions. As shown in ref. [2], simple products of the form $\chi_{ \pm}^{(J)} \bar{\chi}_{ \pm}^{(\bar{J})}$, with $J-\bar{J} \in \mathcal{Z}$ are local. In such a product, the summations over $m$, and $\bar{m}$ are independent, while the summations over $m, \widehat{m}$, and $\bar{m}, \widehat{\bar{m}}$ are correlated. Now we have a complete reversal of the weak coupling situation summarized by Eq.2.5: the new fields preserve the reality condition, but do not preserve the equality between $J$ and $\bar{J}$ quantum numbers. Thus, as already stressed in ref.[2], in the strong coupling regime, we observe a sort of deconfinement of chirality.

\section{The Liouville string}

One may consider two different problems. First, one may build a full-fledged string theory, by coupling, for instance, the above with $26-C$ free fields $\vec{X}$. 
A typical string vertex is of the form $\exp (i \vec{k} \cdot \vec{X}) \chi_{+}^{(J)} \bar{\chi}_{+}^{(\bar{J})}$, where $\vec{k}, J$, and $\bar{J}$ are related so that this is a 1,1 operator. Here obviously, the restriction to real weight is instrumental. Moreover, since one wants the representation of Virasoro algebra to be unitary, one only uses the chi+ fields. This line was already persued with noticable success in refs. [4]. However, the N-point functions seem to be beyond reach at present. Second a simpler problem seems to be tractable, namely, we may proceed as in the construction of topological models just recalled. We consider another copy of the present strongly coupled theory, with central charge $c=26-C$. Since this gives $c=1+6(-s+2)$, we are also at the special values, and the truncation theorems applies to matter as well. This "string theory" has no transverse degree of freedom, and is thus topological. The complete dressed vertex operator is now

$$
\mathcal{V}^{J, \bar{J}}=\chi_{+}^{(J)} \bar{\chi}_{+}^{(\bar{J})} \chi^{\prime(J)} \bar{\chi}^{\prime(\bar{J})}
$$

As in the weak coupling formula, operators relative to matter are distinguished by a prime. The definition of the $\bar{\chi}$ is similar to the above, with an important difference. Clearly, the definition Eq.3.1 of $V_{m,+}^{(J)}$ is not symmetric between $\alpha_{+}$, and $\alpha_{-}$. The truncation theorems also holds if we interchange the two screening charges. We re-establish some symmetry between them by taking the other possible definition for $\bar{\chi}$, namely, we let

$$
\bar{V}_{\bar{m},+}^{(\bar{J})} \equiv \widetilde{\bar{V}}_{\bar{m}-\bar{m}}^{(\bar{J},-\bar{J}-1)}, \quad V_{\bar{m},-}^{(\bar{J})} \equiv V_{\bar{m},(\bar{m})}^{(\bar{J})} .
$$

Our results will then be invariant by complex conjugation provided we exchange $J$ 's and $\bar{J}$ 's. Thus left and right movers are interchanged, which seems to be a sensible requirement. For $J=\bar{J}=0$, we get the new cosmological term

$$
\mathcal{V}^{0,0}=\chi_{+}^{(0)}(z) \bar{\chi}_{+}^{(0)}(\bar{z})
$$

Thus the area element of the strong coupling regime is $\chi_{+}^{(0)}(z) \bar{\chi}_{+}^{(0)}(\bar{z}) d z d \bar{z}$. It is factorized into a simple product of a single $z$ component by a $\bar{z}$ component. From this expression one may compute the string susceptibility using the operator version of the DDK argument developed in ref.[5] for the weak coupling regime. For this we introduce the cosmological constant - so far it was set equal to one. In ref.[5], the weak coupling string susceptibility was 
rederived from the following ansatz

$$
\begin{aligned}
& \left.\widetilde{V}_{m \widehat{m}}^{(J \widehat{J})}\right|_{(\mu)}=\mu^{J+\widehat{J} \alpha_{+} / \alpha_{-}} \mu^{-i p_{0} / \alpha_{-}} V_{m \widehat{m}}^{(J \widehat{J})} \mu^{i p_{0} / \alpha_{-}}
\end{aligned}
$$

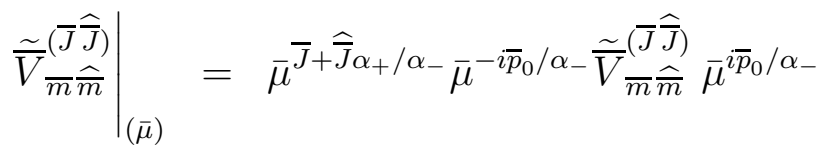

where $p_{0}$ is the zero-mode momentum of the $\vartheta_{L}$ free field. We take a priori two different parameters $\mu$ and $\bar{\mu}$. For the weak coupling case, the previous discussion is immediately recovered with $\mu_{c}=\mu \bar{\mu}$ which is the only parameter that counts. For the strong coupling regime, one substitutes the last equation into Eq.3.4, and its antichiral couterpart. We have defined the $\bar{\chi}$ fields so that taking hermitian conjugate is equivalent to exchanging $J$ 's and $\bar{J}$ 's. We shall thus relate $\mu$ and $\bar{\mu}$ so that the prefactors are connected in the same way. Taking $\mu$ real, this is realized if $\bar{\mu}^{\alpha_{+}}=\mu^{\alpha_{-}}$. Then we have

$$
\left.\left.\chi_{+}^{(J)}\right|_{(\mu)} \bar{\chi}_{+}^{(\bar{J})}\right|_{(\bar{\mu})}=\mu^{-2+Q_{M}\left(\alpha_{-}^{\prime} J+\alpha_{+}^{\prime} \bar{J}\right) / 2} \mu^{-i\left(p_{0} / \alpha_{-}+\bar{p}_{0} / \alpha_{+}\right)} \chi_{+}^{(J)} \bar{\chi}_{+}^{(\bar{J})} \mu^{i\left(p_{0} / \alpha_{-}+\bar{p}_{0} / \alpha_{+}\right)}
$$

For the cosmological term $J=\bar{J}=0$, and the factor becomes $\mu^{-2}$. Thus we conclude that $\mu_{c}=\mu^{2}$. Using the operator definition of correlators (see refs. [5, (7)), and applying Eq.4.5, we get

$$
\left\langle\prod_{\ell} \chi_{+}^{\left(J_{\ell}\right)} \bar{\chi}_{+}^{\left(J_{\ell}\right)}\right\rangle_{\left(\mu_{c}\right)}=\left\langle\prod_{\ell} \chi_{+}^{\left(J_{\ell}^{\prime}\right)} \bar{\chi}_{+}^{\left(J_{\ell}^{\prime}\right)}\right\rangle_{\mu_{c}^{-} \sum_{\ell}\left[1-Q_{M}\left(\alpha_{-}^{\prime} J_{\ell}+\alpha_{+}^{\prime} \bar{J}_{\ell}\right) / 4\right]+(s+2) / 2} .
$$

The last terms emerges when the operators $\mu^{ \pm i\left(p_{0} / \alpha_{-}+\bar{p}_{0} / \alpha_{+}\right)}$hit the left and the right vacuum states (of course due to the background charge). This scaling law is enough to compute] the string susceptibilty. Consider

$$
\mathcal{Z}_{\mu_{c}}(A) \equiv\left\langle\delta\left[\left.\left.\int d z d \bar{z} \chi_{+}^{(0)}\right|_{\left(\mu_{c}\right)} \bar{\chi}_{+}^{(0)}\right|_{\left(\mu_{c}\right)}-A\right]\right\rangle .
$$

One gets

$$
\gamma_{\text {str }}=(2-s) / 2
$$

${ }^{6}$ The previous discussion was actually somewhat different, since $V$ and $\bar{V}$ operators were treated differently. This does not make any difference for the weak coupling regime, but matters at present.

${ }^{7}$ We only consider genus zero. 
The result is real for $c>1(C<25)$, contrary to the continuation of the weak-coupling equation $\gamma_{\text {str }}=2-Q / \alpha_{-}$. Explicitly one has

$$
\left\{\begin{array}{rccc}
s & c & C & \gamma_{\text {str }} \\
1 & 7 & 19 & 1 / 2 \\
0 & 13 & 13 & 1 \\
-1 & 19 & 7 & 3 / 2
\end{array}, \quad\left\{\begin{array}{rccc}
s & c & C & \gamma_{\text {str }} \\
2 & 1 & 25 & 0 \\
-2 & 25 & 1 & 2
\end{array}\right.\right.
$$

The last two are the extreme points of the strong coupling regime. The values at $c=1$, and $c=25$ agree with the weak-coupling formula. The result is always positive, contrary to the weak-coupling regime. At $c=7$, we find the value $\gamma_{\mathrm{str}}=1 / 2$ of branched polymers.

\section{The N-point functions}

We shall concentrate on the N-point function with one incoming and N-1 outgoing legs. The relation between incoming and outgoing momenta is as follows. First in general[ [7] the two-point function of two $\tilde{V}$ fields with spins $J_{1}, \bar{J}_{1}$, and $J_{2}, \bar{J}_{2}$ vanishes unless $J_{1}+J_{2}+1=0$, and $\bar{J}+\bar{J}_{2}+1=0$. Thus conjugation involves the transformation $J \rightarrow-J-1$. Taking account of the exchange between $J$ and $\widehat{J}$, due to complex conjugation, yields the following vertex operator for the conjugate representation:

$$
\mathcal{V}_{\text {conj }}^{J, \bar{J}}=\chi_{+}^{(J)} \bar{\chi}_{+}^{(\bar{J})} \chi_{-}^{(-J-1)} \bar{\chi}_{-}^{(-\bar{J}-1)} .
$$

From the previous discussion we compute that the $\mathrm{N}$ point function depends on the cosmological constant in the following way

$$
\begin{gathered}
\left\langle\mathcal{V}_{\text {conj }}^{J_{1}, \bar{J}_{1}} \mathcal{V}^{J_{2}, \bar{J}_{2}} \ldots \mathcal{V}^{J_{N}, \bar{J}_{N}}\right\rangle_{\mu_{c}} \\
=\mu_{c}^{\left.i Q_{M}\left(\sum_{i=1}^{N}\left[\left(J_{i}+1\right) \alpha_{+}-\left(\bar{J}_{i}+1\right) \alpha_{-}\right)\right]\right) / 4} \mu_{c}^{-(N-2)}\left\langle\mathcal{V}_{\text {conj }}^{J_{1}, \bar{J}_{1}} \mathcal{V}^{J_{2}, \bar{J}_{2}} \ldots \mathcal{V}^{J_{N}, \bar{J}_{N}}\right\rangle .
\end{gathered}
$$

The three-point function was computed in ref. [2]. It is a product of leg factors

$$
L_{J, \bar{J}}=\sqrt{F\left(-Q_{M} \alpha_{+}^{\prime}(J+1 / 2)\right)} \sqrt{F\left(-Q_{M} \alpha_{-}^{\prime}(\bar{J}+1 / 2)\right)} \text { for } \mathcal{V}^{J, \bar{J}}
$$


or

$$
\bar{L}_{J, \bar{J}}=\sqrt{F\left(Q_{M} \alpha_{+}^{\prime}(J+1 / 2)\right)} \sqrt{F\left(Q_{M} \alpha_{-}^{\prime}(\bar{J}+1 / 2)\right)} \text { for } \mathcal{V}_{c o n j}^{J, \bar{J}}
$$

In ref. [2], the three-point function with three vertices $\mathcal{V}^{J_{i}, \bar{J}_{i}}$ was computed. The one with three vertices $\mathcal{V}_{\text {conj }}^{J_{i} \bar{J}_{i}}$ can be obtained by symmetrising $J<->$ $\widehat{J}, J \rightarrow-J-1$, but the one with two vertices $\mathcal{V}^{J_{i}, \bar{J}_{i}}$ and one vertex $\mathcal{V}_{\text {conj }}^{J_{i} \bar{J}_{i}}$ has to be computed again. Remarquably, it still yields the same leg factors ( 2 of one kind, 1 of the other). So, we have the particular three-point function:

$$
\left\langle\mathcal{V}_{\text {conj }}^{J, \bar{J}} \mathcal{V}^{0,0} \mathcal{V}^{J, \bar{J}}\right\rangle_{\mu_{c}}=\mu_{c}^{-Q_{M}\left[\alpha_{-}^{\prime}(2 J+1)+\alpha_{+}^{\prime}(2 \bar{J}+1)\right] / 4-1} \bar{L}_{J, \bar{J}} L_{0,0} L_{J, \bar{J}}
$$

One can check that, at our special values of $C, L_{0,0}=1$. Anyway, for simplicity sake, we renormalize the vertices by these leg factors from now on, without changing notations.

With the new cosmological term, we use the effective action

$$
S_{\mu_{c}}=S_{0}+\mu_{c} \int \mathcal{V}^{0,0}
$$

to generate the N-point functions. Hence

$$
-\frac{\partial}{\partial \mu_{c}}\left\langle\mathcal{V}_{\text {conj }}^{J, \bar{J}} \mathcal{V}^{J, \bar{J}}\right\rangle_{\mu_{c}}=\left\langle\mathcal{V}_{\text {conj }}^{J, \bar{J}} \mathcal{V}^{0,0} \mathcal{V}^{J, \bar{J}}\right\rangle_{\mu_{c}}
$$

and form Eq.5.5

$$
\left\langle\mathcal{V}_{\text {conj }}^{J, \bar{J}} \mathcal{V}^{J, \bar{J}}\right\rangle=\frac{2}{Q_{M}}\left(\alpha_{-}^{\prime}\left(J+\frac{1}{2}\right)+\alpha_{+}^{\prime}\left(\bar{J}+\frac{1}{2}\right)\right)^{-1}
$$

For correlation function with removed external legs, the two-point function is the inverse of the propagator. The correct normalization will turn out to involve an extra $1 / 2$ factor so that the propagator is

$$
P(J, \bar{J})=\frac{Q_{M}}{4}\left(\alpha_{-}^{\prime}\left(J+\frac{1}{2}\right)+\alpha_{+}^{\prime}\left(\bar{J}+\frac{1}{2}\right)\right)
$$

We are now ready to compute the N-point functions $(N>3)$ recursively, or more fundamentally to determine the one-particle irreducible Npoint functions. The first step is the four-point function. The full four-point 
function can be obtained from the three-point function by derivation with respect to $\mu_{c}$ from the three-point function (at least with one zero momentum). It can alternatively be computed by the following decomposition into Feynman diagrams:

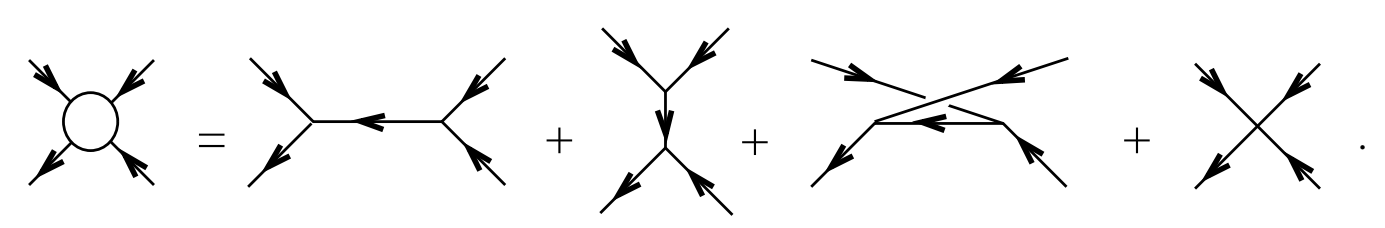

They are oriented diagrams, incoming lines denote $\mathcal{V}^{J, \bar{J}}$ vertices and outcoming lines denote $\mathcal{V}_{\text {conj }}^{J, \bar{J}}$ vertices. The first three diagrams of the r.h.s. can be computed and the difference with the l.h.s. gives the value of the fourth diagram which is the four-point irreducible amplitude. It works similarly for higher order functions, the number of diagrams growing very quickly as usual ( 4 for $\mathrm{N}=4,26$ for $\mathrm{N}=5,236$ for $\mathrm{N}=6,2752$ for $\mathrm{N}=7 \ldots$ ). This has already been done in ref. $[8]$ in the weak coupling case, in the absence of screenings (i.e. with conserved momentum at vertices). We are not generically in this case. The selection rules of our three-point function allows discrete shifts of spin or momentum (see [2]) which amounts, in the Coulomb-gaz approach, to the presence of screening charges for matter. For example, using the short notation $J, \bar{J}$ of the full vertex operator 4.1, in the following diagram

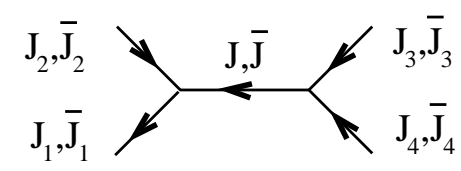

the selection rules of the right hand vertex allows the internal spins $J, \bar{J}$ to be $J_{3}+J_{4}-\nu_{2}, \bar{J}_{3}+\bar{J}_{4}-\bar{\nu}_{2}$, where $\nu_{2}$ and $\bar{\nu}_{2}$ are the number of screenings of the left and right sectors at right hand vertex. $\nu_{2}$ and $\bar{\nu}_{2}$ are positive integers smaller than (or equal to) the total number of screenings $\nu=J_{2}+J_{3}+J_{4}-J_{1}, \bar{\nu}=$ $\bar{J}_{2}+\bar{J}_{3}+\bar{J}_{4}-\bar{J}_{1}$.

We should remember the features specific to the strong coupling regime. The internal states $J, \bar{J}$ are always of the same specific type as the external ones. This is consistent due to the closed braiding and fusing of the $\chi_{ \pm}$ operators. In a propagator, the left and right spins $J$ and $\bar{J}$ are uncorrelated, except that they should only differ by an integer (see [2]). This is also true 
for the number of screenings of the kind $\alpha_{+}$and $\alpha_{-}$which are both in number $\nu$ for the left sector and $\bar{\nu}$ for the right sector. This is in sharp contrast with the weak coupling case.

Let us examine what will happen when we perform the program described above to compute irreducible amplitudes. Beginning from the three-point function essentially equal to 1 , we get the $\mathrm{N}$-point functions by $N-3$ derivations with respect to $\mu_{c}$. We simply get a polynomial of degree $N-3$ in the spins. On the other hand, the computation of a Feynman diagram with fixed internal spins yields a polynomial of degree $N-3$ (at most) in the spins. However we have to sum over all the possible internal spins. For a N-point diagram containing only three-point vertices we have as many possibilities as the number of ways of putting $\nu$ indistinguishable screenings (left sector) and $\bar{\nu}$ other indistinguishable screenings (right sector) on $N-2$ vertices. Computation gives $\left(\begin{array}{l}\nu+N-3 \\ N-3\end{array}\right)\left(\begin{array}{l}\bar{\nu}+N-3 \\ N-3\end{array}\right)$ possibilities (this is 1 for $N=3,(\nu+1)(\bar{\nu}+1)$ for $N=4 \ldots)$. We will therefore get a polynomial of degree $N-3$ in the spins multiplied by this factor depending on $\nu, \bar{\nu}$ (at least for some monomials), in contradiction with the result of the derivation with respect to $\mu_{c}$. This contradiction did not appear in the case without screenings, as this factor is 1 for $\nu=\bar{\nu}=0$. So, we are led to the following new ansatz for the physical amplitudes

$$
\begin{aligned}
& { }^{\text {Phys }} A_{(\nu, \bar{\nu})}^{(N)}\left(\left(J_{1}, \bar{J}_{1}\right),\left(J_{2}, \bar{J}_{2}\right) \ldots\left(J_{N}, \bar{J}_{N}\right)\right) \\
& =\left(\begin{array}{l}
\nu+N-3 \\
N-3
\end{array}\right)\left(\begin{array}{l}
\bar{\nu}+N-3 \\
N-3
\end{array}\right)\left\langle e^{-S_{\mu_{c}}} \int \mathcal{V}_{c o n j}^{J_{1}, \bar{J}_{1}} \mathcal{V}^{J_{2}, \bar{J}_{2}} \ldots \mathcal{V}^{J_{N}, \bar{J}_{N}}\right\rangle
\end{aligned}
$$

such that the derivation is modified in the following way

$$
\begin{gathered}
{ }^{\text {Phys }} A_{(\nu, \bar{\nu})}^{(N+1)}\left(\left(J_{1}, \bar{J}_{1}\right),\left(J_{2}, \bar{J}_{2}\right) \ldots\left(J_{N}, \bar{J}_{N}\right),(0,0)\right) \\
=-\frac{\nu+N-2}{N-2} \frac{\bar{\nu}+N-2}{N-2} \partial_{\mu_{c}}{ }^{P h y s} A_{(\nu, \bar{\nu})}^{(N)}\left(\left(J_{1}, \bar{J}_{1}\right),\left(J_{2}, \bar{J}_{2}\right) \ldots\left(J_{N}, \bar{J}_{N}\right)\right)
\end{gathered}
$$

Let us show explicitely in the case of the four-point function how things can work with this new expression of the physical amplitudes. Eq.5.11 with $N=3$ gives

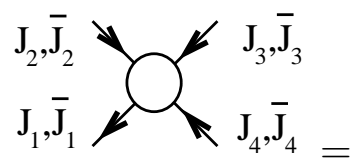




$$
\begin{gathered}
{ }^{\text {Phys }} A_{(\nu, \bar{\nu})}^{(4)}\left(\left(J_{1}, \bar{J}_{1}\right),\left(J_{2}, \bar{J}_{2}\right),\left(J_{3}, \bar{J}_{3}\right),\left(J_{4}, \bar{J}_{4}\right)\right)=(\nu+1)(\bar{\nu}+1) \times \\
\left(1-\frac{Q_{M}}{4}\left[\alpha_{-}^{\prime}\left(J_{1}+J_{2}+J_{3}+J_{4}+1\right)+\alpha_{+}^{\prime}\left(\bar{J}_{1}+\bar{J}_{2}+\bar{J}_{3}+\bar{J}_{4}+1\right)\right]\right)
\end{gathered}
$$

where we have extended the result to non zero $J_{4}, \bar{J}_{4}$ by symmetry. Then, the first three Feynman diagrams can be computed, the sum over the internal momentum yields a prefactor $(\nu+1)(\bar{\nu}+1)$, and the difference with the result above gives the one particule irreducible diagram

$$
\begin{aligned}
& \mathrm{J}_{\mathrm{J}_{4}, \overline{\mathrm{J}}_{4}}^{\mathrm{J}_{3}, \overline{\mathrm{J}}_{3}}={ }^{1 P I} A_{(\nu, \bar{\nu})}^{(4)}\left(\left(J_{1}, \bar{J}_{1}\right),\left(J_{2}, \bar{J}_{2}\right),\left(J_{3}, \bar{J}_{3}\right),\left(J_{4}, \bar{J}_{4}\right)\right) \\
& =(\nu+1)(\bar{\nu}+1) \frac{1}{4}\left((2+s)-\frac{Q_{M}}{2}\left(\alpha_{-}^{\prime} \nu+\alpha_{+}^{\prime} \bar{\nu}\right)\right)
\end{aligned}
$$

At that stage, the question is to know whether we have a check of consistency or not. We notice that, whereas the physical N-point amplitude is symmetric in its $\mathrm{N}$ legs, the Feynman diagrams are not due to the oriented propagators (they are only symmetric in the $N-1$ incoming legs). So, if we want to have symmetric N-point irreducible vertices coming from a to-be-found effective potential, this gives us a very strong check. This is verified for the four-point irreducible function, a little trivially one could say, as it is independent from the momenta. This will no longer be the case for the higher order functions.

So, we followed the same procedure for the five and six-point functions, with the great help of computer (the six-point function involves 236 different diagrams of 12 different ("oriented") topologies, each of them involving six nested sums; the seven-point function would go to 2752 diagrams of 34 different topologies each involving eight nested sums). The results for the irreducible amplitudes are the following:

$$
\begin{gathered}
{ }^{1 P I} A_{(\nu, \bar{\nu})}^{(5)}\left(\left(J_{1}, \bar{J}_{1}\right),\left(J_{2}, \bar{J}_{2}\right),\left(J_{3}, \bar{J}_{3}\right),\left(J_{4}, \bar{J}_{4}\right),\left(J_{5}, \bar{J}_{5}\right)\right) \\
=\left(\begin{array}{c}
\nu+2 \\
2
\end{array}\right)\left(\begin{array}{c}
\bar{\nu}+2 \\
2
\end{array}\right)\left(B^{(5)}(\nu, \bar{\nu})-\sum_{i=1}^{5}\left(P_{i}\right)^{2}\right) \\
\text { where } P_{i}=P\left(J_{i}, \bar{J}_{i}\right)=\frac{Q_{M}}{4}\left[\alpha_{-}^{\prime}\left(J_{i}+\frac{1}{2}\right)+\alpha_{+}^{\prime}\left(\bar{J}_{i}+\frac{1}{2}\right)\right]
\end{gathered}
$$


and $B^{(5)}(\nu, \bar{\nu})$ is the following polynomial of degree 2 in $\nu$ and $\bar{\nu}$ :

$$
\begin{gathered}
B^{(5)}(\nu, \bar{\nu})= \\
2-2 s+s^{2}+\frac{5 a_{-} \nu}{3}+\frac{5 a_{+} \bar{\nu}}{3}-\frac{11 a_{-}^{2} \nu}{12}-\frac{11 a_{+}^{2} \bar{\nu}}{12}+\frac{a_{-}^{2} \nu^{2}}{4}+\frac{a_{+}^{2} \bar{\nu}^{2}}{4}+\frac{10 a_{+} a_{-} \nu \bar{\nu}}{9} \\
\text { with } a_{ \pm}=-Q_{M} \alpha_{ \pm}^{\prime} / 4, \\
{ }^{1 P I} A_{(\nu, \bar{\nu})}^{(6)}\left(\left(J_{1}, \bar{J}_{1}\right),\left(J_{2}, \bar{J}_{2}\right),\left(J_{3}, \bar{J}_{3}\right),\left(J_{4}, \bar{J}_{4}\right),\left(J_{5}, \bar{J}_{5}\right),\left(J_{6}, \bar{J}_{6}\right)\right)= \\
\left({ }_{3}^{\nu+3}\right)\left({ }_{3}^{\bar{\nu}+3}\right)\left[B^{(6)}(\nu, \bar{\nu})-\frac{3}{2}\left((2+s)+\frac{a_{-} \nu}{2}+\frac{a_{+} \bar{\nu}}{2}\right)\left(\sum_{i=1}^{6}\left(P_{i}\right)^{2}\right)\right], \quad(5.16) \\
B^{(6)}(\nu, \bar{\nu})=\frac{1}{16}\left(96-144 a_{+}+140 a_{+}^{2}-46 a_{+}^{3}\right. \\
-144 a_{-}+280 a_{+} a_{-}-138 a_{+}^{2} a_{-} \\
+140 a_{-}^{2}-138 a_{+} a_{-}^{2}-46 a_{-}^{3}+104 a_{-} \nu-18 a_{+} a_{-} \nu+a_{+}^{2} a_{-} \nu-114 a_{-}^{2} \nu \\
+42 a_{+} a_{-}^{2} \nu+49 a_{-}^{3} \nu+28 a_{-}^{2} \nu^{2} \\
+6 a_{+} a_{-}^{2} \nu^{2}-16 a_{-}^{3} \nu^{2}+2 a_{-}^{3} \nu^{3}+104 a_{+} \bar{\nu}-114 a_{+}^{2} \bar{\nu} \\
+49 a_{+}^{3} \bar{\nu}-18 a_{+} a_{-} \bar{\nu}+42 a_{+}^{2} a_{-} \bar{\nu}+a_{+} a_{-}^{2} \bar{\nu} \\
+104 a_{+} a_{-} \nu \bar{\nu}-36 a_{+}^{2} a_{-} \nu \bar{\nu}-36 a_{+} a_{-}^{2} \nu \bar{\nu} \\
\left.+17 a_{+} a_{-}^{2} \nu^{2} \bar{\nu}+28 a_{+}^{2} \bar{\nu}^{2}-16 a_{+}^{3} \bar{\nu}^{2}+6 a_{+}^{2} a_{-} \bar{\nu}^{2}+17 a_{+}^{2} a_{-} \nu \bar{\nu}^{2}+2 a_{+}^{3} \bar{\nu}^{3}\right) .
\end{gathered}
$$

We can now come back to our check of consistency. The external $J_{i}$ 's only appear as squares of the $P_{i}$ 's. This ensures the symmetry under the exchange of incoming and outgoing momenta, so that the sum of Feynman diagrams a priori symmetric in $J_{2}, \ldots J_{N}$, give a vertex also symmetric in $J_{1}, \ldots J_{N}$, as announced. 


\section{Hints for future developments.}

Let us finally review some more ideas we are presently persuing. In the present topological models, both matter and gravity have a background charge. By construction, the stress-energy tensor takes the usual free-field form after Bäcklund transformation. It is thus clearly possible to recombine the Liouville Bäcklund field $\vartheta_{L}$, with its matter counterpart $\vartheta_{M}$ so that the background charge appears in one of the free fields only. For this we let

$$
\vartheta_{L}=-\vec{X} \cdot \vec{\mu}_{L}, \quad \vartheta_{M}=\vec{X} \cdot \vec{\mu}_{M}
$$

where $\vec{X} \equiv(\varphi, X)$, and we introduce the two orthonormalized vectors

$$
\vec{\mu}_{L}=\left(\frac{Q_{L}}{2 \sqrt{2}}, \frac{Q_{M}}{2 \sqrt{2}}\right), \quad \vec{\mu}_{M}=\left(\frac{Q_{M}}{2 \sqrt{2}}, \quad-\frac{Q_{L}}{2 \sqrt{2}}\right) .
$$

Our conventions for $\vec{X}$ coincide with the one of ref. [9], and the present fields are identical apart from the zero-mode spectrum. Let $\vec{X}_{0}$ be the centerof-mass position. It is easy to see that $V_{m, \pm}^{(J)} \propto \exp \left(i m \vec{k}_{ \pm} \cdot \vec{X}_{0}\right)$, and $V_{m, \pm}^{\prime(J)} \propto$ $\exp \left(i m \vec{k}_{ \pm}^{\prime} \cdot \vec{X}_{0}\right)$, where $\vec{k}_{-}=-i Q_{L} \vec{\mu}_{L}, k_{+}=Q_{M} \vec{\mu}_{L}, \vec{k}_{-}^{\prime}=-i Q_{M} \vec{\mu}_{M}$, and $\vec{k}_{+}^{\prime}=Q_{L} \vec{\mu}_{M}$. Remembering the condition $J+m \in \mathcal{Z}_{+}$of eq.3.4, we see that these momenta lie on the lattice generated by the vectors

$$
\begin{array}{cc}
\frac{-i}{2 \sqrt{2}}\left(1, \sqrt{\frac{2-s}{2+s}}\right), & \frac{1}{2 \sqrt{2}}\left(\sqrt{\frac{2+s}{2-s}}, 1\right), \\
\frac{-i}{2 \sqrt{2}}\left(1,-\sqrt{\frac{2+s}{2-s}}\right), & \frac{1}{2 \sqrt{2}}\left(\sqrt{\frac{2-s}{2+s}},-1\right),
\end{array}
$$

which is itself embedded in a four dimensional space with signature 2,2. Thus there may exist a connection between our topological theories and the $N=2$ superstring ${ }^{8}$. Since $Q_{M} \vec{\mu}_{L} / 2-Q_{L} \vec{\mu}_{M} / 2=(0, \sqrt{2})$, it follows that the momenta of the $S U(2)$ generators $\exp \pm i \sqrt{2} X$ belong to the above lattice. Moreover, $k_{+X}=(2-s) / \sqrt{2}, k_{+X}^{\prime}=-(2+s) / \sqrt{2}$. Thus, for integer $s=0$, \pm 1 there are points of the lattice which differ by the momenta $(0, \pm \sqrt{2})$ of the $S U(2)$ generators. Of course, only the $\vec{X}_{0}$ dependence of the $\chi$ fields is simple.

\footnotetext{
${ }^{8}$ See, e.g. ref. $[10]$.
} 
In general Eqs. 3.4 , and 4.1 show that $\mathcal{V}^{J, \bar{J}}$ is a rather involved function of $\vec{X}$ involving momenta of the form $m\left(\vec{k}_{+}+\vec{k}_{-}^{\prime}\right)$. Since that $\left(\vec{k}_{+}+\vec{k}_{-}^{\prime}\right)^{2}=0$, all our on-shell string states are massless, and orthogonal to each other.

Let us turn to a final remark. The redefinition of the cosmological term led us to modify the KPZ formula [1]. On the other hand, in standard studies of the matrix models or KP flows, one first derives $\gamma_{\mathrm{str}}$ and deduces the value of the central charge by assuming that the KPZ formula holds. In this way of thinking, one would start from our formula Eq.4.8 and apply $\mathrm{KPZ}$, which would lead to a different value of the central charge, say $d$. It is easy to see that for $c=1+(-s+2)$ one gets $d=1-6(2-s)^{2} / 2 s$. This is the value of a $2, s$ minimal model! What happens is that in terms of $d$, we have $\gamma_{\mathrm{str}}=(d-1+\sqrt{(d-1)(d-25)}) / 12$, in contrast with the KPZ formula $(d-1-\sqrt{(d-1)(d-25)}) / 12$. Thus our topological theory may be another branch of $d<1$ theories.

Acknowledgements: We are grateful to Ph. Di Franscesco, M. Douglas, V. Kazakov, E. Witten, for very stimulating discussions. This work was partially supported by the E.U. network CHRXCT92005.

\section{References}

[1] J.-L. Gervais, Comm. Math. Phys. 138 (1991) 301.

[2] J.-L. Gervais, J.-F. Roussel, "Solving the strongly coupled 2D gravity: 2. Fractional spin operators and topological three point function", hepth/9403026, Nucl. Phys. to be published.

[3] J.-L. Gervais and A. Neveu, Phys. Lett. B151 (1985) 271.

[4] A. Bilal, J.-L. Gervais, Nucl. Phys. B284 (1987) 397, Phys. Lett. B187 (1987) 39, Nucl. Phys. B293 (1987) 1, Nucl. Phys. B295 [FS21] (1988) 277.

[5] J.-L. Gervais, Nucl. Phys. B391 (1993) 287.

[6] J.-L. Gervais, J. Schnittger, Phys. Lett. B315 (1993) 258; "Continous spins in 2D gravity: chiral vertex operators and construction of the local Liouville field", hep-th/9405136. 
[7] E. Cremmer, J.-L. Gervais, J.-F. Roussel, Nucl. Phys. B413 (1994) 244; Comm. Math. Phys. 161 (1994) 597.

[8] P. Di Francesco, D. Kutasov, Nucl. Phys. B375 (1992) 119.

[9] E. Witten, Nucl. Phys. B373 (1992) 187.

[10] H. Ooguri, C. Vafa, Nucl. Phys. B361 (1991) 469.

[11] V. Knizhnik, A. Polyakov, A.A. Zamolodchikov, Mod. Phys. Lett. A3 (1988) 819. 
This figure "fig1-1.png" is available in "png" format from: http://arxiv.org/ps/hep-th/9408076v1 